\title{
Variational derivation of two-component Camassa-Holm shallow water system
}

\author{
Delia IONESCU-KRUSE \\ Institute of Mathematics of the Romanian Academy, \\ P.O. Box 1-764, RO-014700, Bucharest, Romania \\ E-mail: Delia.lonescu@imar.ro
}

\begin{abstract}
By a variational approach in the Lagrangian formalism, we derive the nonlinear integrable two-component Camassa-Holm system (11). We show that the two-component Camassa-Holm system (1) with the plus sign arises as an approximation to the Euler equations of hydrodynamics for propagation of irrotational shallow water waves over a flat bed. The Lagrangian used in the variational derivation is not a metric.
\end{abstract}

Keywords: two-component Camassa-Holm system, shallow water waves, variational methods.

AMS Subject Classification: 35Q53, 76B15, 70G75

\section{Introduction}

A two-component generalization of the peakon Camassa-Holm equation is given by

$$
\left\{\begin{array}{l}
u_{t}+3 u u_{x}-u_{t x x}-2 u_{x} u_{x x}-u u_{x x x} \pm H H_{x}=0 \\
H_{t}+(H u)_{x}=0
\end{array}\right.
$$

with $x \in \mathbf{R}, t \in \mathbf{R}, u(x, t) \in \mathbf{R}, H(x, t) \in \mathbf{R}$. Subscripts here, and later, denote partial derivatives. Obviously, for $H=0$, the system (11) reduces to the peakon equation derived by Camassa and Holm [4 (for alternative derivations within the shallow water regime see also Refs. 25], [13, 22, [10]).

The choice of the minus sign is often considered in (10). This system appears originally in [32, where it is shown that most integrable bi-Hamiltonian systems are governed by tri-Hamiltonian structures. Then, by recombining the Hamiltonian operators one is lead to integrable hierarchies, one of this integrable hierarchy including the system (10) with minus sign. Alternative derivations of 
the system (10) with the minus sign are provided in [34, [27, 6], 16]. In [6] is established an explicit reciprocal transformation between (1), called here the 2-CH system, and the first negative flow of the AKNS hierarchy. Thus, solutions of the 2-CH system are obtained from solutions of the first negative flow of the AKNS hierarchy, among them the peakon and multi-kink solutions. In [16] it is also given a Lax pair formulation of the system (11). So far, there is no physical interpretation of the system (11) with the choice of the minus sign.

For the choice of the plus sign in (1), the system may be regarded as a model of shallow water waves. In [9] this system is derived from the GreenNaghdi equation 1 [17 by using expansions of the variable in terms of physical parameters. There it is also noticed that, with the asymptotic limits $u \rightarrow 0$ and $H \rightarrow 1$ as $|x| \rightarrow \infty$, the peakon solution is absent among the solitary waves solutions of (11) with the plus sign. A modified two-component CamassaHolm system is proposed in 21]. This modified system, derived in 21] as semidirect-product Euler-Poincaré equations 20 with the Lagrangian given by a $H^{1}$ metric in $u$ and $H^{1}$ metric in $(H-1)$, allows singular solutions, but may not be integrable.

The mathematical properties of the system (11) have been studied further in many works. Its analytical properties such as well-posedness and wave breaking were studied in [14, 9], 18] and others; more about its geometric properties see in [15. For investigations of the traveling wave solutions we refer to [9], 30], 29.

The purpose of the present paper is to derive by a variational approach in the Lagrangian formalism, the two-component Camassa-Holm shallow water system, that is, the system (10) with the plus sign. Starting from a general dimensionless version of the irrotational water wave equations, we introduce the two fundamental parameters $\epsilon$ and $\delta$, associated with amplitude and with wave length. The shallow water approximation is obtained by requiring $\delta \rightarrow 0$, for arbitrary fixed $\epsilon$. In this regime, the leading-order problem reduces to a system of two equations on the surface elevation and the horizontal component of the velocity, that is, the system (12), or (14) in view of the notation (13). These equations are the so-called classical shallow water equations (see, for example, 35]). We are looking for a higher-order correction to the classical shallow water equations. The second equation in (14), which can be interpreted as a continuity equation for the free surface elevation, is exactly the second equation of the system (10). The first equation of the system (1) with plus sign, will be an Euler-Lagrange equation yielding the critical points of an action (functional) in the space of path, within the Lagrangian formalism. Firstly, we approximate the kinetic energy at the free surface. We consider the parameter $\epsilon$ such that $\epsilon^{3}$ and $\epsilon^{4}$ contributions in the kinetic energy at the free surface (19) can be neglected. In the Eulerian picture, the Lagrangian is defined as the approximated kinetic energy at the free surface (20) minus the potential energy calculated with respect to the undisturbed water level (21). In the Lagrangian

\footnotetext{
1 For more background information on the Green-Naghdi equations, see, for example, the discussion in the recent book 8 .
} 
formalism, the Lagrangian will be obtained from the Lagrangian in the Eulerian picture, taking also into account the second equation of the system (10), see (33). This Lagrangian is not a metric. To the order of our approximation, we get that the non-dimensional horizontal velocity of the water $u$ and the non-dimensional free upper surface $H$, satisfy the Camassa-Holm shallow water system (11) with the plus sign, see Theorem 1. We also observe that, the Camassa-Holm system (11) with the minus sign is obtained if instead of the Lagrangian (32) we consider the total energy at the free surface, that is, the approximated kinetic energy at the free surface (20) plus the potential energy calculated with respect to the undisturbed water level (21). This Lagrangian is a metric, namely, a $H^{1}$ metric in $u$ and $L^{2}$ metric in $(H-1)$. We point out that (1) with the minus sign can be regarded as geodesic equations on the semidirect product of $\operatorname{Diff}(\mathbf{R})$ with $\mathcal{F}(\mathbf{R})$ (see also 21]).

The derivation of the Camassa-Holm shallow water system (11) with the plus sign is made under the assumption of irrotational flow. There are circumstances in which this assumption is well justified but there are cases where it is inappropriate. Tidal flow is a well-known example when constant vorticity flow is an appropriate model (see [12]; for a discussion of the physical relevance of flows with constant vorticity see also [7]). The derivation of a two-component system modelling shallow water waves in the constant vorticity case, by introducing of suitable scalings and by truncating asymptotic expansions of the quantities to appropriate order, is given in [23]. The derivation of such a model by a variational approach in the Lagrangian formalism will be presented in a future paper.

\section{Nondimensionalisation of the governing equations for water waves. Classical shallow water equations}

The two-dimensional gravity water waves moving over an irrotational flow are described by the following boundary value problem:

$$
\begin{gathered}
u_{t}+u u_{x}+v u_{z}=-p_{x} \\
v_{t}+u v_{x}+v v_{z}=-p_{z}-g \\
u_{x}+v_{z}=0 \\
u_{z}-v_{x}=0 \\
v=\eta_{t}+u \eta_{x} \text { on } z=h_{0}+\eta(x, t) \\
v=0 \text { on } z=0 \\
p=p_{0} \text { on } z=h_{0}+\eta(x, t)
\end{gathered}
$$

where $(u(x, z, t), v(x, z, t))$ is the velocity field of the water - no motion takes place in the $y$-direction, $p(x, z, t)$ denotes the pressure, $g$ is the constant gravitational acceleration, $p_{0}$ being the constant atmospheric pressure. The water 
moves in a domain with a free upper surface at $z=h_{0}+\eta(x, t)$, for a constant $h_{0}>0$, and a flat bottom at $z=0$. The undisturbed water surface is $z=h_{0}$ and $\eta(x, t)$ is the displacement of the free surface from the undisturbed state. Euler's equations (EEs) are the appropriate equations of motion for gravity water waves, see 24]. Another realistic assumption for gravity water wave problem is the incompressibility, that is, the density $\rho$ is constant (see [26]), which implies the equation of mass conservation (MC). We set the constant water density $\rho=1$. The idealization of irrotational flow is physically relevant in the absence of non-uniform currents in the water. The zero vorticity flow is characterized by the additional equation (IC). The kinematic boundary conditions (KBCs) express the fact that the same particles always form the free-water surface and that the fluid is assumed to be bounded below by a hard horizontal bed $z=0$. The dynamic boundary condition (DBC) expresses the fact that on the free surface the pressure is equal to the constant atmospheric pressure denoted $p_{0}$.

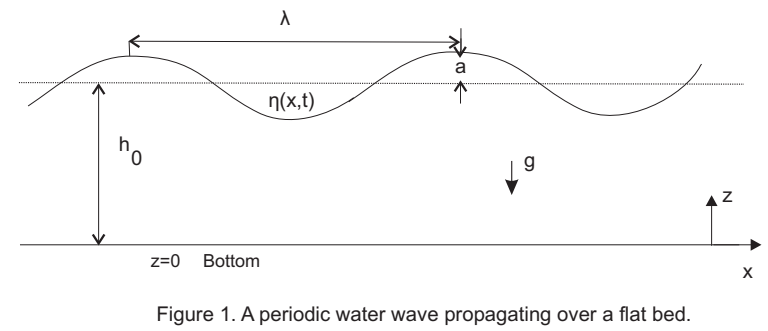

We non-dimensionalize the set of equations (2) using the undisturbed depth of the water $h_{0}$, as the vertical scale, a typical wavelength $\lambda$, as the horizontal scale, and a typical amplitude of the surface wave $a$ (see Figure 1). Thus, we define the set of non-dimensional variables (for more details see [24]):

$$
\begin{aligned}
x \mapsto \lambda x, \quad z \mapsto h_{0} z, \quad \eta \mapsto a \eta, \quad t \mapsto \frac{\lambda}{\sqrt{g h_{0}}} t, \\
u \mapsto \sqrt{g h_{0}} u, \quad v \mapsto h_{0} \frac{\sqrt{g h_{0}}}{\lambda} v, \\
p \mapsto p_{0}+g h_{0}(1-z)+g h_{0} p,
\end{aligned}
$$

where, to avoid new notations, we have used the same symbols for the nondimensional variables $x, z, \eta, t, u, v, p$ on the right-hand side. Therefore, in the 
non-dimensional variables (3), the water-wave problem (2) becomes:

$$
\begin{array}{cc}
u_{t}+u u_{x}+v u_{z}=-p_{x} & \\
\delta^{2}\left(v_{t}+u v_{x}+v v_{z}\right)=-p_{z} & \\
u_{x}+v_{z}=0 & \\
u_{z}-\delta^{2} v_{x}=0 & \text { on } z=1+\epsilon \eta(x, t) \\
v=\epsilon\left(\eta_{t}+u \eta_{x}\right) & \text { on } z=1+\epsilon \eta(x, t) \\
p=\epsilon \eta & \text { on } z=0 \\
v=0 &
\end{array}
$$

where we have introduced the amplitude parameter $\epsilon=\frac{a}{h_{0}}$ and the shallowness parameter $\delta=\frac{h_{0}}{\lambda}$.

The shallow water approximation is obtained by requiring $\delta \rightarrow 0$, for arbitrary fixed $\epsilon$. For $\delta=0$, the leading-order equations become:

$$
\begin{array}{cc}
u_{t}+u u_{x}+v u_{z}=-p_{x} & \\
p_{z}=0 & \\
u_{x}+v_{z}=0 & \\
u_{z}=0 & \\
v=\epsilon\left(\eta_{t}+u \eta_{x}\right) & \text { on } z=1+\epsilon \eta(x, t) \\
p=\epsilon \eta(x, t) & \text { on } z=1+\epsilon \eta(x, t) \\
v=0 & \text { on } z=0
\end{array}
$$

From the second equation and the forth equation in (5), we get that $u$ and $p$ do not depend on $z$. Thus,

$$
u=u(x, t),
$$

and because $p=\epsilon \eta(x, t)$ on $z=1+\epsilon \eta(x, t)$, we get

$$
p=\epsilon \eta(x, t) .
$$

Taking into account (6) and the third equation in (5) we obtain

$$
v=-z u_{x}+g(x, t),
$$

$g(x, t)$ being an arbitrary function. Further, by the last equation in (5), we have

$$
v=-z u_{x}
$$

With (6) and (7) in view, the first equation in (5) can be written as

$$
u_{t}+u u_{x}+\epsilon \eta_{x}=0 .
$$

From (9), the fifth equation in (5) becomes

$$
\epsilon \eta_{t}+\epsilon u \eta_{x}+u_{x}+\epsilon \eta u_{x}=0 .
$$

Hence, for $\delta=0$, the system which describes the evolution of the surface waves is

$$
\left\{\begin{array}{l}
u_{t}+u u_{x}+\epsilon \eta_{x}=0 \\
\epsilon \eta_{t}+[(1+\epsilon \eta) u]_{x}=0,
\end{array}\right.
$$


the other two variables $p$ and $v$ are expressed function of $\eta$ and $u$, see (77), respectively (9). In the study of the full governing equations for water waves, the relations between the free surface, the fluid velocity and the pressure are more subtle. Various properties of the pressure and the velocity beneath Stokes waves and deep-water Stokes waves are proved, by methods relying upon harmonic and superharmonic functions, in [11, respectively [19.

If we denote by

$$
H(x, t):=1+\epsilon \eta(x, t)
$$

then, the system of equations (12) becomes

$$
\left\{\begin{array}{l}
u_{t}+u u_{x}+H_{x}=0 \\
H_{t}+(H u)_{x}=0
\end{array}\right.
$$

The set of hyperbolic partial differential equations (14) are the so-called classical shallow water equations (see, for example, 35]). The equations (14) can be written in Hamiltonian form relative to a symplectic structure introduced by Manin 28. The second Hamiltonian structure for the system (14) was obtained by Cavalcante and McKean [5]. In fact, the system (14), as a particular case of the system of polytropic gas equations in $1+1$, i.e. in one spatial and one temporal dimension, is Hamiltonian with respect to three distinct Hamiltonian structures [31]. These Hamiltonian structures are compatible and thus, the system of equations (14) is completely integrable 33. The infinite sequence of integrals of motion (the conserved quantities) that the Hamiltonian structures give rise to was found much earlier by Benney [3. The equations (14) provide a good approximation to the exact solution of the water-wave problem; for a rigorous justification with a precise control of the estimated error see [2].

\section{Approximation procedure and variational derivation of the two-component Camassa-Holm shallow water system}

By an interplay of small-parameter expansions and variational methods we derive the two-component Camassa-Holm shallow water equations.

The two important parameters $\epsilon$ and $\delta$ that arise in water-waves theories in the forms $\epsilon$ and $\delta^{2}$, are used to define various approximations of the governing equations and the boundary conditions. The role of $\delta$ independent of $\epsilon$ is useful in the description of arbitrary amplitude shallow water waves, that is, $\delta \rightarrow 0$, $\epsilon$ fixed. The small-amplitude shallow water waves are obtained in the limits $\epsilon \rightarrow 0$ and $\delta \rightarrow 0$.

We observe in (4) that on $z=1+\epsilon \eta$ both $v$ and $p$ are proportional to $\epsilon$, this being consistent with the fact that as $\epsilon \rightarrow 0$ we must have $v \rightarrow 0$ and

$p \rightarrow 0$ (with no disturbance the free surface becomes a horizontal surface on which $v=p=0$ ). For consistence, one requires that $u$ is proportional to $\epsilon$ too. $\epsilon$ is also called the nonlinearity parameter. Indeed, we see that the terms $u u_{x}$ and $\epsilon \eta u$ from (12), are proportional with $\epsilon^{2}$ and by neglecting these terms, the 
system (12) reduces to the linear system

$$
\left\{\begin{array}{l}
u_{t}+\epsilon \eta_{x}=0 \\
\epsilon \eta_{t}+u_{x}=0
\end{array}\right.
$$

Therefore, $\eta(x, t)$ satisfies the linear wave equation

$$
\eta_{t t}-\eta_{x x}=0
$$

By choosing the right-going wave of (16), that is,

$$
\eta(x, t)=f(x-t)
$$

we obtain the solution

$$
u=\epsilon \eta(x, t)+\text { const, } v=-\epsilon z \eta_{x}(x, t), p=\epsilon \eta(x, t) .
$$

We are looking for a higher-order correction to the classical shallow water equations (12), or (14) in view of the notation (13). We observe that the second equation in (14) is exactly the second equation of the two-component CamassaHolm shallow water system (11). In order to get the two-component CamassaHolm shallow water system (11) with the plus sign, instead of making asymptotic expansions in the equations of motion, we approximate the kinetic energy at the free surface and we use the variational methods in the Lagrangian formalism.

Taking into account the components (6) and (9) of the velocity field, the kinetic energy has at the free surface $z=1+\epsilon \eta(x, t)$ the expression

$$
E_{c}(u, \eta)=\frac{1}{2} \int_{-\infty}^{\infty}\left[u^{2}+(1+\epsilon \eta)^{2} u_{x}^{2}\right] d x
$$

As we saw above, the non-dimensional $u$ is proportional to $\epsilon$. We consider the parameter $\epsilon$ such that $\epsilon^{3}$ and $\epsilon^{4}$ contributions in the right hand side of (19) can be neglected. Thus, we approximate the kinetic energy at the free surface by

$$
E_{c} \approx \frac{1}{2} \int_{-\infty}^{\infty}\left[u^{2}+u_{x}^{2}\right] d x=: E_{c}(u) .
$$

We require in (20) that $u(x, t)$ and $u_{x}(x, t)$ decay rapidly at \pm infinity, at any instant $t$.

In non-dimensional variables, with $\rho$ and $g$ settled at 1 , we define the gravitational potential energy at the free surface $z=1+\epsilon \eta(x, t)$, gained by the fluid parcel when it is vertically displaced from its undisturbed position with $\epsilon \eta(x, t)$, by

$$
\begin{gathered}
E_{p}(\eta)=\int_{-\infty}^{\infty}\left(\int_{0}^{1+\epsilon \eta}(z-1) d z\right) d x=\frac{1}{2} \int_{-\infty}^{\infty}(\epsilon \eta)^{2} d x \\
\stackrel{13}{=} \frac{1}{2} \int_{-\infty}^{\infty}(H-1)^{2} d x=: E_{p}(H)
\end{gathered}
$$


We require in (21) that $H(x, t) \rightarrow 1$ as $x \rightarrow \pm \infty$, at any instant $t$.

The second equation in (14) was derived by replacing in the free-surface kinematic boundary condition, that is, the fifth equation in (5), the vertical velocity $v$ obtained from the mass conservation equation, that is, the third equation in (5), for an horizontal velocity $u$ given by (6) and taking also into account that the rigid bottom is impenetrable. The Lagrangian interpretation of the free-surface kinematic boundary condition is that a particle on the surface always stays on the surface. The free-surface kinematic boundary condition is a transport equation, the free surface is advected, or Lie transported (in the geometry literature), by the fluid flow. Otherwise, the second equation in (14) can be interpreted as a continuity equation for the free surface, the same as the continuity equation for the mass density in the compressible fluids. With the formula of the Lie derivative of a 1-form along a vector field in view (see, for example, 11 Section 2.2.), the second equation in (14) expresses the fact that the 1-form $\mathrm{H}(x, t):=H(x, t) d x$ is advected, or Lie transported, by the vector field $\mathrm{u}(x, t):=u(x, t) \partial_{x}$, that is,

$$
\frac{\partial \mathrm{H}}{\partial t}+\mathrm{L}_{\mathrm{u}} \mathrm{H}=0
$$

where $\mathrm{L}_{\mathrm{u}}$ denotes the Lie derivative with respect to the vector field $\mathrm{u}$.

In the Lagrangian formalism one focuses the attention on the motion of each individual particle of the mechanical system. We denote by $M$ the ambient space whose points are supposed to represent the particles at $t=0$. A diffeomorphism of $M$ represents the rearrangement of the particles with respect to their initial positions. The set of all diffeomorphisms, denoted $\operatorname{Diff}(M)$, can be regarded (at least formally) as a Lie group. The motion of the mechanical system is described by a time-dependent family of orientation-preserving diffeomorphisms $\gamma(\cdot, t) \in$ $\operatorname{Diff}(M)$. For a particle initially located at $X$, the velocity at time $t$ is

$$
\gamma_{t}(X, t):=\frac{\partial \gamma(X, t)}{\partial t}
$$

this being the material velocity used in the Lagrangian description. The spatial velocity, used in the Eulerian description, is the flow velocity

$$
u(x, t):=\gamma_{t}(X, t),
$$

at the location $x=\gamma(X, t)$, at time $t$, that is,

$$
u(\cdot, t)=\gamma_{t} \circ \gamma^{-1} .
$$

In the Lagrangian description, the velocity phase space is the tangent bundle $T \operatorname{Diff}(M)$. In the Eulerian description, the spatial velocity is in the tangent space at the identity $I d$ of $\operatorname{Diff}(M)$, that is, it is an element of the Lie algebra of $\operatorname{Diff}(M)$.

In our problem, $\mathrm{u}(x, t)$ can be regarded as a time-dependent vector field on $\mathbf{R}$, that is, it belongs to the Lie algebra of $\operatorname{Diff}(\mathbf{R})$. Thus, in the Lagrangian 
formalism of our problem we take $M=\mathbf{R}$ and add the technical assumption that the smooth functions defined on $\mathbf{R}$ with value in $\mathbf{R}$ vanish rapidly at $\pm \infty$ together with as many derivatives as necessary. The configuration space of our problem is $\operatorname{Diff}(\mathbf{R})$. For a motion $\gamma(\cdot, t) \in \operatorname{Diff}(\mathbf{R})$ we have its Lagrangian velocity given by (23) and its Eulerian velocity given by (25). $\gamma$ is the flow of the time-dependent vector field $u$.

The other unknown of our problem is $H(x, t)$, which for a fixed $t$ can be regarded as a real function on $\mathbf{R}, H(\cdot, t) \in \mathcal{F}(\mathbf{R})$. We settle that the evolution equation of $H(x, t)$ is the second equation in (14) which can be written in the form (22). The second equation in (14) and the equation (22) are equations in the Eulerian picture. With the aid of the pull back map $\gamma^{*}$, we can write the Lagrangian form of the equation (22), that is,

$$
\gamma^{*}\left(\frac{\partial \mathrm{H}}{\partial t}+\mathrm{L}_{\mathrm{u}} \mathrm{H}\right)=0
$$

We use further the interpretation of the Lie derivative of a time-dependent 1form along a time-dependent vector field in terms of the flow of the vector field (see, for example, 1], Section 2), and we get the equation

$$
\frac{d}{d t}\left[\gamma^{*}(\mathrm{H})\right]=\gamma^{*}\left(\mathrm{~L}_{\mathrm{u}} \mathrm{H}\right)+\gamma^{*}\left(\frac{\partial \mathrm{H}}{\partial t}\right) \stackrel{2 \sigma]}{=} 0 .
$$

We denote this time invariant 1-form in the reference configuration by

$$
\mathrm{H}_{0}:=\gamma^{*}(\mathrm{H}), \quad \mathrm{H}_{0}(X, t)=\mathrm{H}_{0}(X, 0) .
$$

By the definiton of the pull back map (see, for example, 1, Section 2), we get between the components of the 1 -forms $\mathrm{H}_{0}(X, t):=H_{0}(X, t) d X$ and $\mathrm{H}(x, t):=$ $H(x, t) d x$ the following relation

$$
H_{0}=(H \circ \gamma) J_{\gamma}
$$

where $J_{\gamma}:=\frac{\partial \gamma}{\partial X}$ is the Jacobian of $\gamma$, or,

$$
H=\left(H_{0} \circ \gamma^{-1}\right) J_{\gamma^{-1}} .
$$

In Lagrangian description, the equation of motion is the equation satisfied by a critical point of a certain functional $\mathfrak{a}(\gamma)$, called the action,

$$
\mathfrak{a}(\gamma):=\int_{0}^{T} \mathcal{L}\left(\gamma, \gamma_{t}\right) d t
$$

defined on all paths $\{\gamma(\cdot, t), t \in[0, T]\}$ in $\operatorname{Diff}(M)$, having fixed endpoints. $\mathcal{L}$ is a scalar function defined on $T \operatorname{Diff}(M)$, called Lagrangian.

The Lagrangian for our problem will be obtained by transporting the Lagrangian from the Eulerian picture, which by (20) and (21) is defined by

$$
\mathfrak{L}(u, H)=E_{c}(u)-E_{p}(H)=\frac{1}{2} \int_{-\infty}^{\infty}\left[u^{2}+u_{x}^{2}-(H-1)^{2}\right] d x,
$$


to all tangent spaces $T \operatorname{Diff}(\mathbf{R})$, this transport being made taking into account (25) and (30).

For each function $H_{0} \in \mathcal{F}(\mathbf{R})$ independent of time, we define the Lagrangian $\mathcal{L}_{H_{0}}: T \operatorname{Diff}(\mathbf{R}) \rightarrow \mathbf{R}$ by

$$
\mathcal{L}_{H_{0}}\left(\gamma, \gamma_{t}\right):=\frac{1}{2} \int_{-\infty}^{\infty}\left\{\left(\gamma_{t} \circ \gamma^{-1}\right)^{2}+\left[\partial_{x}\left(\gamma_{t} \circ \gamma^{-1}\right)\right]^{2}-\left[\left(H_{0} \circ \gamma^{-1}\right) J_{\gamma^{-1}}-1\right]^{2}\right\} d x
$$

The Lagrangian $\mathcal{L}_{H_{0}}$ depends smoothly on $H_{0}$ and it is right invariant under the action of the subgroup

$$
\operatorname{Diff}(\mathbf{R})_{H_{0}}=\left\{\psi \in \operatorname{Diff}(\mathbf{R}) \mid\left(H_{0} \circ \psi^{-1}\right) J_{\psi^{-1}}=H_{0}\right\}
$$

that is, if we replace the path $\gamma(t, \cdot)$ by $\gamma(t, \cdot) \circ \psi(\cdot)$, for a fixed time-independent $\psi$ in $\operatorname{Diff}(\mathbf{R})_{H_{0}}$, then $\mathcal{L}_{H_{0}}$ is unchanged.

The action on a path $\gamma(t, \cdot), t \in[0, T]$, in $\operatorname{Diff}(\mathbf{R})$ is

$$
\mathfrak{a}(\gamma):=\int_{0}^{T} \mathcal{L}_{H_{0}}\left(\gamma, \gamma_{t}\right) d t
$$

The critical points of the action (35) in the space of paths with fixed endpoints, satisfy

$$
\left.\frac{d}{d \varepsilon} \mathfrak{a}(\gamma+\varepsilon \varphi)\right|_{\varepsilon=0}=0
$$

for every path $\varphi(t, \cdot), t \in[0, T]$, in $\operatorname{Diff}(\mathbf{R})$ with endpoints at zero, that is, $\varphi(0, \cdot)=0=\varphi(T, \cdot)$ and such that $\gamma+\varepsilon \varphi$ is a small variation of $\gamma$ on $\operatorname{Diff}(\mathbf{R})$. Taking into account (33) and (35), the condition (36) becomes

$$
\begin{aligned}
\int_{0}^{T} \int_{-\infty}^{\infty} & \left\{\left.\left(\gamma_{t} \circ \gamma^{-1}\right) \frac{d}{d \varepsilon}\right|_{\varepsilon=0}\left[\left(\gamma_{t}+\varepsilon \varphi_{t}\right) \circ(\gamma+\varepsilon \varphi)^{-1}\right]\right. \\
& +\left.\partial_{x}\left(\gamma_{t} \circ \gamma^{-1}\right) \frac{d}{d \varepsilon}\right|_{\varepsilon=0}\left[\partial_{x}\left(\left(\gamma_{t}+\varepsilon \varphi_{t}\right) \circ(\gamma+\varepsilon \varphi)^{-1}\right)\right] \\
& -\left.\left(H_{0} \circ \gamma^{-1}\right) J_{\gamma^{-1}}^{2} \frac{d}{d \varepsilon}\right|_{\varepsilon=0}\left[H_{0} \circ(\gamma+\varepsilon \varphi)^{-1}\right] \\
& -\left.\left(H_{0} \circ \gamma^{-1}\right)^{2} J_{\gamma^{-1}} \frac{d}{d \varepsilon}\right|_{\varepsilon=0}\left[J_{(\gamma+\varepsilon \varphi)^{-1}}\right] \\
& +\left.\left(J_{\gamma^{-1}}\right) \frac{d}{d \varepsilon}\right|_{\varepsilon=0}\left[H_{0} \circ(\gamma+\varepsilon \varphi)^{-1}\right] \\
& \left.+\left.\left(H_{0} \circ \gamma^{-1}\right) \frac{d}{d \varepsilon}\right|_{\varepsilon=0}\left[J_{(\gamma+\varepsilon \varphi)^{-1}}\right]\right\} d x d t=0 .
\end{aligned}
$$

Let us now proceed to the calculations of the terms in (37). For the first two terms in (37) see, for example, 22].

Firstly, differentiating with respect to $\varepsilon$ the identity

$$
(\gamma+\varepsilon \varphi) \circ(\gamma+\varepsilon \varphi)^{-1}=I d
$$


one gets

$$
\left.\frac{d}{d \varepsilon}\right|_{\varepsilon=0}(\gamma+\varepsilon \varphi)^{-1}=-\frac{\varphi \circ \gamma^{-1}}{\gamma_{x} \circ \gamma^{-1}}
$$

We also have

$$
\begin{aligned}
& \partial_{x}\left(\gamma_{t} \circ \gamma^{-1}\right)=\left(\gamma_{t x} \circ \gamma^{-1}\right) \partial_{x}\left(\gamma^{-1}\right)=\frac{\gamma_{t x} \circ \gamma^{-1}}{\gamma_{x} \circ \gamma^{-1}} \\
& \partial_{x}\left(\varphi \circ \gamma^{-1}\right)=\left(\varphi_{x} \circ \gamma^{-1}\right) \partial_{x}\left(\gamma^{-1}\right)=\frac{\varphi_{x} \circ \gamma^{-1}}{\gamma_{x} \circ \gamma^{-1}} \\
& \partial_{t}\left(\varphi \circ \gamma^{-1}\right)=\varphi_{t} \circ \gamma^{-1}+\left(\varphi_{x} \circ \gamma^{-1}\right) \partial_{t}\left(\gamma^{-1}\right) \\
& =\varphi_{t} \circ \gamma^{-1}-\left(\gamma_{t} \circ \gamma^{-1}\right) \partial_{x}\left(\varphi \circ \gamma^{-1}\right) \text {, } \\
& \partial_{x}^{2}\left(\gamma_{t} \circ \gamma^{-1}\right)=\partial_{x}\left(\frac{\gamma_{t x} \circ \gamma^{-1}}{\gamma_{x} \circ \gamma^{-1}}\right) \\
& =\frac{\left(\gamma_{t x x} \circ \gamma^{-1}\right)}{\left(\gamma_{x} \circ \gamma^{-1}\right)^{2}}-\frac{\left(\gamma_{t x} \circ \gamma^{-1}\right)\left(\gamma_{x x} \circ \gamma^{-1}\right)}{\left(\gamma_{x} \circ \gamma^{-1}\right)^{3}} \text {, } \\
& \left.\frac{d}{d \varepsilon}\right|_{\varepsilon=0}\left[\partial_{x}(\gamma+\varepsilon \varphi)^{-1}\right]=\left.\frac{d}{d \varepsilon}\right|_{\varepsilon=0}\left[\frac{1}{\left(\gamma_{x}+\varepsilon \varphi_{x}\right) \circ(\gamma+\varepsilon \varphi)^{-1}}\right] \\
& =-\frac{\partial_{x}\left(\varphi \circ \gamma^{-1}\right)}{\gamma_{x} \circ \gamma^{-1}}+\frac{\gamma_{x x} \circ \gamma^{-1}}{\left(\gamma_{x} \circ \gamma^{-1}\right)^{3}}\left(\varphi \circ \gamma^{-1}\right) \text {, } \\
& \left.\frac{d}{d \varepsilon}\right|_{\varepsilon=0}\left[\left(\gamma_{t x}+\varepsilon \varphi_{t x}\right) \circ(\gamma+\varepsilon \varphi)^{-1}\right]=\varphi_{t x} \circ \gamma^{-1}-\left(\gamma_{t x x} \circ \gamma^{-1}\right) \frac{\varphi \circ \gamma^{-1}}{\gamma_{x} \circ \gamma^{-1}}
\end{aligned}
$$

Taking into account (39) - 450 obtain

$$
\begin{aligned}
\left.\frac{d}{d \varepsilon}\right|_{\varepsilon=0}\left[\left(\gamma_{t}+\varepsilon \varphi_{t}\right) \circ(\gamma+\varepsilon \varphi)^{-1}\right] & =\partial_{t}\left(\varphi \circ \gamma^{-1}\right)+\left(\gamma_{t} \circ \gamma^{-1}\right) \partial_{x}\left(\varphi \circ \gamma^{-1}\right) \\
& -\left(\varphi \circ \gamma^{-1}\right) \partial_{x}\left(\gamma_{t} \circ \gamma^{-1}\right) \\
\left.\frac{d}{d \varepsilon}\right|_{\varepsilon=0}\left[\partial_{x}\left(\left(\gamma_{t}+\varepsilon \varphi_{t}\right) \circ(\gamma+\varepsilon \varphi)^{-1}\right)\right]= & \partial_{t x}\left(\varphi \circ \gamma^{-1}\right)+\left(\gamma_{t} \circ \gamma^{-1}\right) \partial_{x}^{2}\left(\varphi \circ \gamma^{-1}\right) \\
& -\left[\partial_{x}^{2}\left(\gamma_{t} \circ \gamma^{-1}\right)\right]\left(\varphi \circ \gamma^{-1}\right) \\
\left.\frac{d}{d \varepsilon}\right|_{\varepsilon=0}\left[H_{0} \circ(\gamma+\varepsilon \varphi)^{-1}\right]= & \left.\left(H_{0_{x}} \circ \gamma^{-1}\right) \frac{d}{d \varepsilon}\right|_{\varepsilon=0}(\gamma+\varepsilon \varphi)^{-1} \\
= & -\left(\varphi \circ \gamma^{-1}\right) \partial_{x}\left(H_{0} \circ \gamma^{-1}\right)
\end{aligned}
$$




$$
\begin{aligned}
\left.\frac{d}{d \varepsilon}\right|_{\varepsilon=0}\left[J_{\left.(\gamma+\varepsilon \varphi)^{-1}\right]}\right. & =\left.\frac{d}{d \varepsilon}\right|_{\varepsilon=0}\left[\partial_{x}(\gamma+\varepsilon \varphi)^{-1}\right] \\
& =-\frac{\partial_{x}\left(\varphi \circ \gamma^{-1}\right)}{\gamma_{x} \circ \gamma^{-1}}+\frac{\gamma_{x x} \circ \gamma^{-1}}{\left(\gamma_{x} \circ \gamma^{-1}\right)^{3}}\left(\varphi \circ \gamma^{-1}\right) \\
& =-\left(J_{\gamma^{-1}}\right) \partial_{x}\left(\varphi \circ \gamma^{-1}\right)-\partial_{x}\left(J_{\gamma^{-1}}\right)\left(\varphi \circ \gamma^{-1}\right) .
\end{aligned}
$$

Thus, with (46)-(49) in view, the condition (37) becomes

$$
\begin{aligned}
\int_{0}^{T} \int_{-\infty}^{\infty} \quad & \left\{u\left[\partial_{t}\left(\varphi \circ \gamma^{-1}\right)+u \partial_{x}\left(\varphi \circ \gamma^{-1}\right)-\left(\varphi \circ \gamma^{-1}\right) u_{x}\right]\right. \\
& +u_{x}\left[\partial_{t x}\left(\varphi \circ \gamma^{-1}\right)+u \partial_{x}^{2}\left(\varphi \circ \gamma^{-1}\right)-\left(\varphi \circ \gamma^{-1}\right) u_{x x}\right] \\
& +H H_{x}\left(\varphi \circ \gamma^{-1}\right)+H^{2} \partial_{x}\left(\varphi \circ \gamma^{-1}\right) \\
& \left.-H_{x}\left(\varphi \circ \gamma^{-1}\right)-H \partial_{x}\left(\varphi \circ \gamma^{-1}\right)\right\} d x d t=0
\end{aligned}
$$

where $u=\gamma_{t} \circ \gamma^{-1}$ and $H=\left(H_{0} \circ \gamma^{-1}\right) J_{\gamma^{-1}}$. We integrate by parts with respect to $t$ and $x$ in the above formula, we take into account that $u \rightarrow 0, u_{x} \rightarrow 0$, $H \rightarrow 1$ at $\pm \infty$ and $\varphi$ has endpoints at zero, and finally we get

$$
-\int_{0}^{T} \int_{-\infty}^{\infty}\left(\varphi \circ \gamma^{-1}\right)\left[u_{t}+3 u u_{x}-u_{t x x}-2 u_{x} u_{x x}-u u_{x x x}+H H_{x}\right] d x d t=0
$$

Therefore, to the order of our approximation, we proved:

Theorem 1. For an irrotational shallow water flow, the non-dimensional horizontal velocity of the water $u(x, t)$ and the non-dimensional free upper surface $H(x, t)=1+\epsilon \eta(x, t)$, satisfy the Camassa-Holm shallow water system (1) with the plus sign.

Remark. We observe that, if instead of the Lagrangian (32) we consider the $H^{1}$ metric in $u$ and $L^{2}$ metric in $(H-1)$, that is,

$$
\frac{1}{2} \int_{-\infty}^{\infty}\left[u^{2}+u_{x}^{2}+(H-1)^{2}\right] d x
$$

by transporting this metric to all tangent spaces $T \operatorname{Diff}(\mathbf{R})$, the transport being made taking into account (25) and (30), we get, for each function $H_{0} \in \mathcal{F}(\mathbf{R})$ independent of time, $\mathcal{E}_{H_{0}}: T \operatorname{Diff}(\mathbf{R}) \rightarrow \mathbf{R}$ defined by

$$
\mathcal{E}_{H_{0}}\left(\gamma, \gamma_{t}\right):=\frac{1}{2} \int_{-\infty}^{\infty}\left\{\left(\gamma_{t} \circ \gamma^{-1}\right)^{2}+\left[\partial_{x}\left(\gamma_{t} \circ \gamma^{-1}\right)\right]^{2}+\left[\left(H_{0} \circ \gamma^{-1}\right) J_{\gamma^{-1}}-1\right]^{2}\right\} d x
$$

$\mathcal{E}_{H_{0}}$ depends smoothly on $H_{0}$ and its right invariant under the action of the subgroup (34). The critical points of the action

$$
\int_{0}^{T} \mathcal{E}_{H_{0}}\left(\gamma, \gamma_{t}\right) d t
$$


in the space of paths with fixed endpoints, will satisfy

$$
-\int_{0}^{T} \int_{-\infty}^{\infty}\left(\varphi \circ \gamma^{-1}\right)\left[u_{t}+3 u u_{x}-u_{t x x}-2 u_{x} u_{x x}-u u_{x x x}-H H_{x}\right] d x d t=0
$$

Thus, the functions $u(x, t)$ and $H(x, t)$ will fulfill the Camassa-Holm system (11) with the sign minus. We point out that (10) with the minus sign can be regarded as geodesic equations on the semidirect product of $\operatorname{Diff}(\mathbf{R})$ with $\mathcal{F}(\mathbf{R})$ (see also [21]).

\section{References}

[1] R. Abraham and J. E. Marsden, "Foundations of Mechanics", BenjaminCummings, London ISBN 0-8053-0102-X, 1978.

[2] B. Alvarez-Samaniego and D. Lannes, Large time existence for $3 D$ waterwaves and asymptotics, Invent. Math. 171 (2008), 485-541.

[3] D. J. Benney, Some properties of long non-linear waves, Studies Appl. Math. 52 (1973) 45-50.

[4] R. Camassa and D. D. Holm, An integrable shallow water equation with peaked solitons, Phys. Rev. Letters 71 (1993), 1661-1664.

[5] J. Cavalcante and H. P. McKean, The Classical Shallow Water Equations: Symplectic Geometry, Physica 4D(1982), 253-260.

[6] M. Chen, S.-Q. Liu, Y. Zhang, A two-component generalization of the Camassa-Holm Equation and its Solutions, Lett. Math. Phys. 75 (2006), $1-15$.

[7] A. Constantin, Two-dimensionality of gravity water flows of constant nonzero vorticity beneath a surface wave train, Eur. J. Mech. B Fluids 30 (2011), 12-16.

[8] A. Constantin, "Nonlinear Water Waves with Applications to WaveCurrent Interactions and Tsunamis", CBMS-NSF Conference Series in Applied Mathematics, Vol. 81, SIAM, Philadelphia, 2011.

[9] A. Constantin, R. I. Ivanov, On an integrable two-componet Camassa-Holm shallow water system, Phys. Lett. A 372 (2008), 7129-7132.

[10] A. Constantin and D. Lannes, The hydrodynamical relevance of the Camassa-Holm and Degasperis-Procesi equations, Arch. Ration. Mech. Anal. 192 (2009), 165-186.

[11] A. Constantin and W. Strauss, Pressure beneath a Stokes wave, Comm. Pure Appl. Math. 53 (2010), 533-557. 
3 Approximation procedure and variational derivation of the two-component Camassa-Holm shallow water system 14

[12] T. A. Da Silva and D. H. Peregrine, Steep, steady surface waves on water of finite depth with constant vorticity, J. Fluid. Mech., 195 (1988), 281-302.

[13] H. R. Dullin, G. Gottwald and D. D. Holm, Camassa-Holm, Korteweg-de Vries-5 other asymptotically equivalent equations for shallow water waves, Fluid Dyn. Res. 90 (2003), 73-95.

[14] J. Escher, O. Lechtenfeld and Z. Yin, Well-posedness and blow-up phenomena for the 2-component Camassa-Holm equation, Discrete Contin. Dyn. Syst. 19 (2007), 493-513.

[15] J. Escher, M. Kohlmann, J. Lenells, The geometry of the two-components Camassa-Holm and Degasperis-Procesi equations, J. Geom. Phys. 61 (2011), 436-452.

[16] G. Falqui, On a Camassa-Holm type equation with two dependent variables, J. Phys. A: Math. Gen 39 (2006), 327-342.

[17] A. Green and P. Naghdi, A derivation of equations for wave propagation in water of variable depth, J. Fluid Mech. 78 (1976), 237-246.

[18] C. Guan and Z. Yin, Global existance and blow-up phenomena for an integrable two-component Camassa-Holm shallow water system, J. Differential Equations 248 (2010), 2003-2014.

[19] D. Henry, Pressure in a deep-water Stokes wave, J. Math. Fluid Mech. 13 (2011), 251-257.

[20] D. D. Holm, J. E. Marsden and T. Ratiu, The Euler Poincare Equations and Semidirect Products with Applications to Continuum Theories, Advances in Mathematics 137 (1998), 1-81.

[21] D. D. Holm, L. O. Naraigh and C. Tronci, Singular solutions of a modified two-component Camassa-Holm equation, Phys. Rev. 79 (2009), no. 1, 016601, 13 pp.

[22] D. Ionescu-Kruse, Variational derivation of the Camassa-Holm shallow water equation, J. Nonlinear Math. Phys. 14 (2007), 303-312.

[23] R. I. Ivanov, Two-component integrable systems modelling shallow water waves: the constant vorticity case, Wave Motion 46 (2009), 389-396.

[24] R. S. Johnson, "A Modern Introduction to the Mathematical Theory of Water Waves", Cambridge Univeristy Press, 1997.

[25] R. S. Johnson, Camassa-Holm, Korteweg-de Vries and related models for water waves, J. Fluid Mech. 455 (2002), 63-82.

[26] J. Lighthill, "Waves in Fluids", Cambridge University Press, 2001. 
[27] S. Q. Liu and J. E. Zhang, Deformations of semisimple bihamiltonian structures of hydrodynamic type, J. Geom. Phys. 54 (2005), 427-453.

[28] Yu. I. Manin, Algebraic Aspects of Nonlinear Differential Equations, Sov. Prob. Mat. 11 (1978), 5-152.

[29] K. Mohajer, A note on traveling wave solutions to the two-component Camassa-Holm equation, J. Nonliner Math. Phys. 16 (2009), 117-125.

[30] O. Mustafa, On smooth traveling waves of an integrable two-component Camassa-Holm shallow water system, Wave Motion 46 (2009), 397-402.

[31] Y. Nutku, On a new class of completely integrable nonlinear wave equations. II. Multi-Hamiltonian structure, J. Math. Phys. 28 (1987), 2579-2585.

[32] P. J. Olver and P. Rosenau, Tri-Hamiltonian duality between solitons and solitary-wave solutions having compact support, Phys. Rev. E 53 (1996), 1900-1906.

[33] P.J. Olver and Y. Nutku, Hamiltonian structures for systems of hyperbolic conservation laws, J. Math. Phys. 29 (1988), 1610.

[34] A. B. Shabat and L. Martinez Alonso, On the prolongation of a hierarchy of hydrodynamic chains, in: A. B. Shabat et al. (Eds.), New Trends in integrability and partial solvability, Proceedings of the NATO Advanced Research Workshop, Cadiz, Spain 2002, NATO Science Series, Kluwer Academic Publisher, Dordrecht, 2004, 263-280.

[35] J. J. Stoker, "Water Waves: The Mathematical Theory with Applications", Wiley-Interscience New-York, 1992. 